\title{
Fault Detection in Chemical Processes Using Discriminant Analysis and Control Chart
}

\author{
Xudong Pei*, Yoshiyuki YAMASHITA**, \\ Masatoshi YoshidA and Shigeru MATSUMOTO \\ Department of Chemical Engineering, Tohoku University, \\ 6-6-07, Aramaki Aoba, Sendai-shi, Miyagi 980-8579, Japan
}

Keywords: Process Monitoring, Fault Detection, Control Charts, Fisher Discriminant Analysis

This paper presents a fault detection methodology based on the Fisher discriminant analysis (FDA) and individuals control charts (XmR control charts). As the first step, FDA is used to find the optimal discriminant direction between the normal operation data and the fault data. In the next step, XmR control charts on the discriminant direction are used to monitor the process. To reduce the amount of false alarms, we also used a variable selection technique based on the contribution plot of FDA. The performance of the proposed technique is demonstrated through application to the monitoring of the Tennessee Eastman challenge process.

\section{Introduction}

We are all in agreement that the technique of process monitoring plays an important role in the operation of chemical processes. The work of monitoring includes fault detection, fault identification and diagnosis. For successful monitoring of any process, it is important to detect faults as early as possible. And then one can make action to reconstruct or recover the faulty process.

Data-driven techniques have become an active research field for monitoring chemical processes, not only because the process data are ready to be used in many process systems, but also because the techniques do not need any rigorous models. It is often difficult or costly to obtain mathematical models for complex chemical processes, which limits the application of model-based techniques for the monitoring.

Among many data-driven techniques, statistical process monitoring (SPM) methods have been widely used. Conventional SPM methods assume that the measured values are independent and normally distributed. Typical control charts, such as Shewhart control charts (Shewhart, 1931), cumulative sum (CUSUM) control charts (Page, 1954), and exponentially weighted moving average (EWMA) control charts (Roberts, 1959), are well established for monitoring univariate

Received on April 9, 2007; accepted on August 14, 2007. Correspondence concerning this article should be addressed to Y. Yamashita (E-mail address: yama_pse@cc.tuat.ac.jp).

* Present address: Beijing Institute of Technology, No. 5, Zhongguancun Nandajie Haidian District, Beijing 100081, P.R. China.

** Present address: Tokyo University of Agriculture and Technology, 2-24-16, Naka-cho, Koganei-shi, Tokyo 184-8588, Japan. systems. Originally the mean and the variance were separately monitored on the two control charts. Integration of the mean and the variance on a control chart was first proposed by Gan (1997). Recently several similar control charts have been proposed, such as the Max chart (Chen and Cheng, 1998), alternate variables chart (Spiring and Cheng, 1998), Max-CUSUM chart, SS-CUSUM chart (Thaga, 2003), SSEWMA chart (Xie, 1999), Max-EWMA chart (Chen et al., 2001) and EMWA-SC chart (Chen et al., 2004). A review of single variables control charts can be found in a recent article (Cheng and Thaga, 2006).

These control charts are fast, accurate and intuitive to monitor univariate variation with control limits. However, chemical processes have a large number of variables, and the assumptions for the variables are often invalid due to their dynamic, multivariate and nonlinear natures. It is also difficult to choose the appropriate variables for detection or identification of a fault. As the result, the application of the conventional univariate SPM methods on chemical processes is limited.

Multivariate control charts were developed to overcome these inconvenience. The first multivariate control chart is the Hotelling's $T^{2}$ statistic (Hotelling, 1947) which uses one common statistic to plot multivariate observations by combining their dispersions and means. After this work, many multivariate control charts have been proposed, such as the multivariate CUSUM charts (Crosier, 1988; Pignatiello and Runger, 1990), multivariate EWMA charts (Lowry and Woodall, 1992), Alternate variables chart (Spiring and Cheng, 1998), multivariate Max-CUSUM chart and multivariate Max chart (Thaga, 2003). When the number of variables in a process becomes large, a lot 
of redundant information among these variables easily makes these multivariate control charts impractical. Another approach to multivariate monitoring is using $T^{2}$ statistic and $Q$ statistic based on principal component analysis (PCA), which was originally proposed by Kresta et al. (1991) and has been successfully applied to many processes. The $T^{2}$ statistic measures the variation of principal components, and the $Q$ statistic measures the variation of nonprincipal components. Later on, many improvements have been studied, such as the multiway PCA for batch processes (Nomikos and MacGregor, 1994), dynamic PCA introducing dynamic behavior into the PCA model (Ku et al., 1995), multiscale PCA based on wavelet analysis (Bakshi, 1998), recursive PCA (Li et al., 2000), dynamic PCA for batch monitoring with time-lagged windows (Chen and Liu, 2002), kernel PCA for nonlinear process monitoring (Cho et al., 2005; Lee et al., 2004; Schölkopf et al., 1998), and Robust multiscale PCA (Wang and Romagnoli, 2005). Qin (2003) reviewed several fault detection indices associated with $T^{2}$ statistic and $Q$ statistic and compared the reconstruction-based approach and the contribution-based approach with simulation and industrial examples. Fisher discriminant analysis (FDA) has been also used for process monitoring as a dimensionality reduction technique. Different from the PCA-based methods which use only the information in the normal data, the FDA-based methods include all the information by maximally separating fault data from the normal data during the process monitoring. Chiang et al. (2000) compared the miss-classification rate of FDA, PCA and partial least squares (PLS) for the Tennessee Eastman process, and showed that the FDA was better than PLS and PCA. He et al. (2005) compared the result of fault diagnosis by using the contribution plots based on the FDA and PCA models, and showed that the FDA was superior to the PCA.

In the present work, a new statistical process monitoring method based on the FDA and control charts is proposed. The FDA is used to search the optimal onedimensional discriminant direction between the fault data and the normal data. Individuals control charts (XmR charts), one of the most popular control charts, are used to monitor the fault data on the optimal discriminant direction. Contribution plot based on the optimal discriminant direction from FDA is also used to improve its performance. The proposed method is applied to the Tennessee Eastman (TE) process. The proposed method is evaluated and compared with the $T^{2}$ and $Q$ statistical methods based on the PCA in terms of the average run length (ARL).

\section{Method}

In this section, the basics of the FDA, XmR and key variable selection are shortly summarized at first. And then a method for fault detection based on the FDA and $\mathrm{XmR}$ charts is introduced.

\subsection{Fisher Discriminant Analysis}

Fisher discriminant analysis is a linear dimensionality reduction technique to find a direction for which data classes are optimally separated. The optimal discriminant direction is determined by maximizing the scatter between the classes while minimizing the scatter within classes. In this subsection, the FDA is shortly summarized.

Given a set of $n d$-dimensional samples $x_{1}, x_{2}, \cdots$, $x_{n}$. Let's divide the samples into two subsets, $\mathcal{X}_{1}$ and $\mathcal{X}_{2}$, each of which has $n_{1}$ and $n_{2}$ samples $\left(n_{1}+n_{2}=n\right)$. The $d$-dimensional sample mean of each classes is defined as

$$
\boldsymbol{m}_{i}=\frac{1}{n_{i}} \sum_{\boldsymbol{x} \in \mathcal{X}_{i}} \boldsymbol{x} \quad i=1,2
$$

If there is a one-dimensional projection direction $\boldsymbol{w}$, the projection of the sample vector $\boldsymbol{x}_{j}$ on $\boldsymbol{w}$ is given by

$$
y_{j}=\boldsymbol{w}^{T} \boldsymbol{x}_{j} \quad j=1,2, \cdots, n
$$

The corresponding set of $n$ projections $y_{1}, y_{2}, \cdots, y_{n}$ are divided into the subsets $\mathcal{Y}_{1}$ and $\mathcal{Y}_{2}$. The corresponding means for the projections are given $\tilde{m}_{1}$ and $\tilde{m}_{2}$.

$$
\tilde{m}_{i}=\boldsymbol{w}^{T} \boldsymbol{m}_{i} \quad i=1,2
$$

The scatter for projected samples is defined as

$$
\tilde{s}_{i}^{2}=\frac{1}{n_{i}} \sum_{y \in \mathcal{Y}_{i}}\left(y-\tilde{m}_{i}\right)^{2} \quad i=1,2
$$

Fisher discriminant analysis employs the projection direction $\boldsymbol{w}$ which maximises the following objective function

$$
J(\boldsymbol{w})=\frac{\left(\tilde{m}_{1}-\tilde{m}_{2}\right)^{2}}{\tilde{s}_{1}^{2}+\tilde{s}_{2}^{2}}
$$

The separation of the projected means obeys

$$
\begin{aligned}
\left(\tilde{m}_{1}-\tilde{m}_{2}\right)^{2} & =\left(\boldsymbol{w}^{T} \boldsymbol{m}_{1}-\boldsymbol{w}^{T} \boldsymbol{m}_{2}\right)^{2} \\
& =\boldsymbol{w}^{T}\left(\boldsymbol{m}_{1}-\boldsymbol{m}_{2}\right)\left(\boldsymbol{m}_{1}-\boldsymbol{m}_{2}\right)^{T} \boldsymbol{w} \\
& =\boldsymbol{w}^{T} S_{\mathrm{b}} \boldsymbol{w}
\end{aligned}
$$

where $S_{\mathrm{b}}$ is the between-class scatter matrix defined as

$$
S_{\mathrm{b}}=\left(\boldsymbol{m}_{1}-\boldsymbol{m}_{2}\right)\left(\boldsymbol{m}_{1}-\boldsymbol{m}_{2}\right)^{T}
$$

Similarly, the scatters give 


$$
\tilde{s}_{1}^{2}+\tilde{s}_{2}^{2}=\boldsymbol{w}^{T} S_{\mathrm{w}} \boldsymbol{w}
$$

where $S_{\mathrm{w}}$ is the within-class scatter matrix defined as

$$
S_{\mathrm{w}}=\sum_{i=1}^{2}\left(\frac{1}{n_{i}} \sum_{x \in \mathcal{X}_{i}}\left(x-m_{i}\right)\left(x-m_{i}\right)^{T}\right)
$$

In terms of $S_{\mathrm{b}}$ and $S_{\mathrm{w}}$, the Fisher criterion function can be rewritten as

$$
J(\boldsymbol{w})=\frac{\boldsymbol{w}^{T} S_{\mathrm{b}} \boldsymbol{w}}{\boldsymbol{w}^{T} S_{\mathrm{w}} \boldsymbol{w}}
$$

To maximise the function $J(\cdot)$, the direction $w$ is identical to the eigenvector corresponding to the largest eigenvalue of the generalized eigenvalue problem;

$$
S_{\mathrm{b}} \boldsymbol{w}=\lambda S_{\mathrm{w}} \boldsymbol{w}
$$

If $S_{\mathrm{w}}$ is nonsingular, Eq. (11) can be rewritten as the conventional eigenvalue problem.

$$
S_{\mathrm{w}}^{-1} S_{\mathrm{b}} \boldsymbol{w}=\lambda \boldsymbol{w}
$$

For the purpose of fault detection, two classes of data sets are considered corresponding to the normal data and the current data. In this study, the sequence of two continuous samples in the current data are considered as the current data. For only the two data samples, it is not meaningful to calculate their scatter. So we simplify the calculation of $S_{\mathrm{w}}$ as

$$
S_{\mathrm{w}}=\frac{1}{n_{1}} \sum_{\boldsymbol{x} \in \mathcal{X}_{1}}\left(\boldsymbol{x}-\boldsymbol{m}_{1}\right)\left(\boldsymbol{x}-\boldsymbol{m}_{1}\right)^{T}
$$

where $\mathcal{X}_{1}$ denotes the normal subset.

\subsection{Individuals control charts}

Control charts have been used for many years around the world, and it has greatly contributed to improve the quality of many processes. The individual and moving range chart, or XmR chart, is one of the most popular control charts.

Given a set of $n$ samples $x_{1}, x_{2}, \cdots, x_{i}, \cdots, x_{n}$, the moving range is defined as the absolute difference between two successive samples,

$$
M R_{i}=\left|x_{i}-x_{i-1}\right|
$$

which indicates possible shifts or changes in the process between the samples.

Based on this moving range, two kinds of methods are well known in computing control-limits for
$\mathrm{XmR}$ charts. One is to use the average of the moving range and set limits at a distance of multiplying the average by a scaling factor 2.660 from the center line. The other is to use the median of the moving range and set the limits at a distance of multiplying the median by a scaling factor 3.145 from the center line. In this work, the average of the moving range is used.

Let $\bar{x}$ be the average of the samples and $\overline{M R}$ be the average of the moving range, then upper control limit UCL and lower control limit LCL are defined as

$$
\begin{aligned}
\mathrm{UCL} & =\bar{x}+2.660 \overline{M R} \\
\mathrm{LCL} & =\bar{x}-2.660 \overline{M R}
\end{aligned}
$$

where the XmR chart constant, 2.660, can be found in Smith (2001) or any other basic SPC textbooks. If a sample $x_{i}$ satisfies LCL $\leq x_{i} \leq \mathrm{UCL}$, the sample is considered within the normal range.

\subsection{Variable selection}

By using selected subset of variables, the classification result often becomes better than the result by using all the variables. Many methods have been proposed for variable selection, such as the contribution plot of $T^{2}$ or $Q$ statistics (Miller et al., 1998), genetic algorithm/Fisher discriminant analysis (Chiang and Pell, 2004), and so on. In this work, contribution plots based on the optimal discriminant direction $w$ from FDA is used to select key variables.

Suppose $\boldsymbol{w}=\left[w_{1}, w_{2}, \cdots, w_{d}\right]$ be the optimal discriminant direction between the normal data set and the fault data set calculated by FDA. The element $w_{i}$ determines the average contribution of the $i$-th variable to the separation of the two data sets. Important variables for the separation can be selected based on the value $\left|w_{i}\right|$. In this work, the variables whose contribution is larger than $0.1 w_{\max }$ are selected. Where $w_{\max }$ is the maximum value of the contribution.

$$
w_{\max }=\max \left(\left|w_{1}\right|,\left|w_{2}\right|, \cdots,\left|w_{d}\right|\right)
$$

\subsection{Fault detection methodology}

By combining FDA and XmR charts, the following fault-detection methodology is proposed here. The methodology includes two steps: preliminary detection and verified detection. In the first step, FDA is applied to normal data and each class of abnormal data to find optimal separation direction and $\mathrm{XmR}$ is used to monitor the result. In the next step, FDA is recalculated on the reduced variables, which are selected based on the contribution plots, and validate the detected faults.

1.4.1 Preliminary detection In the first step, optimal discriminant direction between current data and normal data is computed based on the Fisher criteria. On the calculated discriminant direction, control limits LCL and UCL for XmR charts of the projected data 


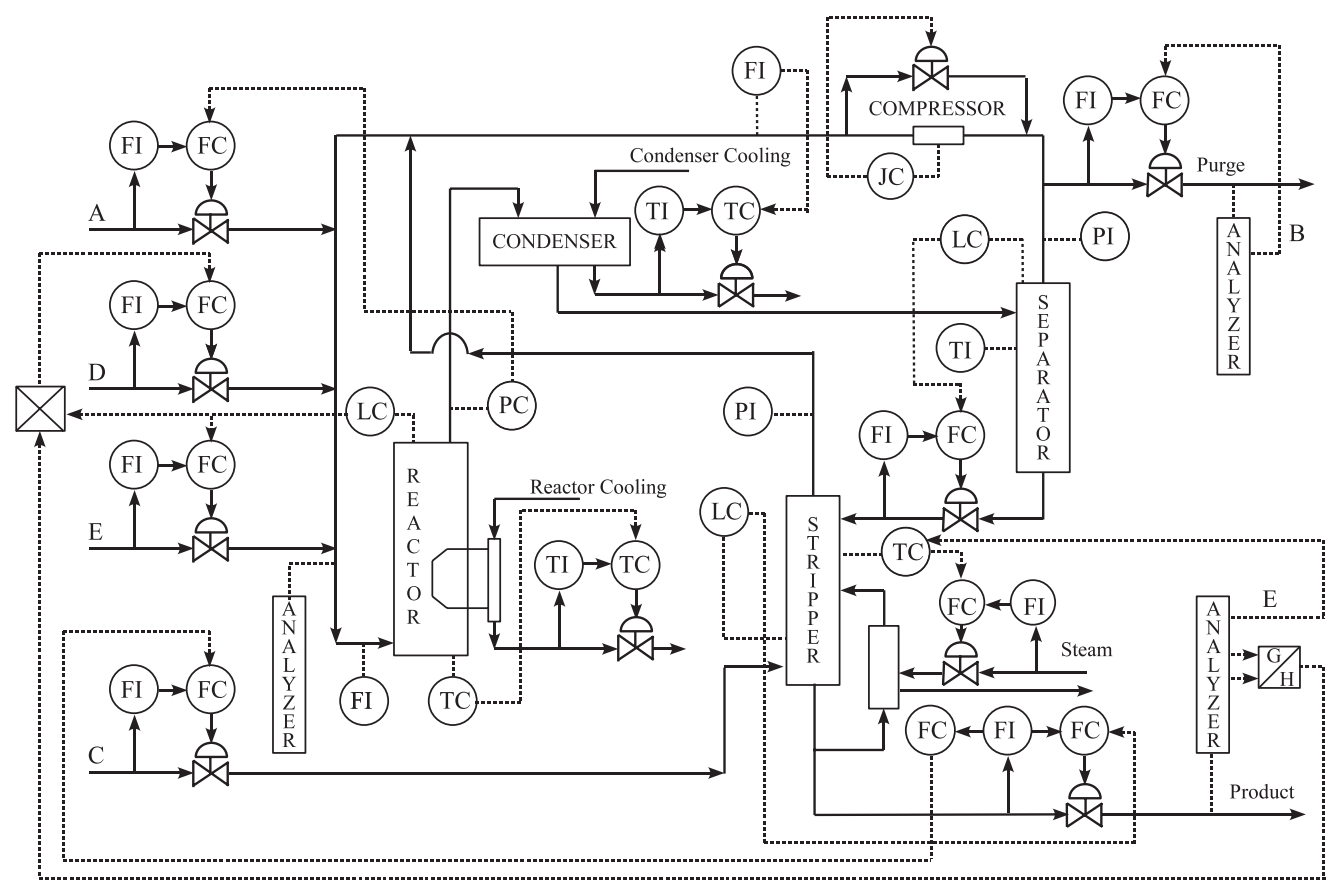

Fig. 1 The Tennessee Eastman process with base controllers

are calculated. A fault is considered to be detected if all the projections of the current data do not satisfy the following condition;

$$
\mathrm{LCL}_{1} \leq x_{1,2}^{1} \leq \mathrm{UCL}_{1}
$$

where $x_{1,2}^{1}$ is the projections of current data on $\boldsymbol{w}_{1}$.

If a fault is not detected in this step, the system is considered within the normal operation and terminate the detection procedure. To deal with several normal operating conditions, normal data set must be prepared for each normal operation.

1.4.2 Verifying detection If a fault is detected in the first step, the fault is verified in the second step. In the second step, at first, key variables are selected by using the contribution plot based on $\boldsymbol{w}_{1}$. By using only the selected variables, optimal discriminant direction $\boldsymbol{w}_{2}$ between the current data and the normal data is computed based on the Fischer criteria. On this optimal direction, control limits LCL2 and UCL2 for XmR charts of the projected data are considered.

If the following condition is not satisfied, the process is considered in an abnormal condition and the detected fault in the first step is considered to be verified.

$$
\mathrm{LCL}_{2} \leq x_{1,2}^{2} \leq \mathrm{UCL}_{2}
$$

where $x_{1,2}^{2}$ is the projection of the current sample of the selected variable on $\boldsymbol{w}_{2}$. If this condition is satisfied, the detected fault is canceled in the verification step.

\section{Application}

\subsection{Target plant}

The proposed method is applied to the Tennessee Eastman (TE) plant (Downs and Vogel, 1993; McAvoy and Ye, 1994). Figure 1 shows a schematic diagram of the TE plant, which includes five major units: a reactor, a product condenser, a vapor-liquid separator, a recycle compressor and a product stripper. It converts four source materials, A, C, D and E, into two products, $\mathrm{G}$ and $\mathrm{H}$.

In this work, twenty two continuous measurement variables listed in Table $\mathbf{1}$ are used for monitoring. Variable names in right-side column show where and what to be measured. For example, XMEAS(1) measures the feed flowrate of the source material A Thirteen disturbances designed in the TE simulator are listed in Table 2. In this table, the first 7 disturbances are step changes, the next 5 are random changes, and the last one is a slow drift. Five runs of simulated process data were generated for each disturbance as well as the normal case. Each run contains 100 normal samples and 100 abnormal samples. The sampling interval is $3 \mathrm{~min}$.

\subsection{Variable selection}

Let's consider a time series of normal operation as an example. Among 100 samples, 98 samples are used as normal data and the last two samples are used as current data. Projections on discriminant directions $\boldsymbol{w}_{1}$ and $\boldsymbol{w}_{2}$ are calculated with corresponding control 
Table 1 Continuous process measurements

\begin{tabular}{ll}
\hline XMEAS(1) & A feed flowrate \\
XMEAS(2) & D feed flowrate \\
XMEAS(3) & E feed flowrate \\
XMEAS $(4)$ & A and C feed flowrate \\
XMEAS(5) & Recycle flowrate \\
XMEAS $(6)$ & Reactor feed rate \\
XMEAS(7) & Reactor pressure \\
XMEAS $(8)$ & Reactor level \\
XMEAS $(9)$ & Reactor temperature \\
XMEAS $(10)$ & Purge rate \\
XMEAS $(11)$ & Product separator temperature \\
XMEAS $(12)$ & Product separator level \\
XMEAS $(13)$ & Product separator pressure \\
XMEAS $(14)$ & Product separator underflow \\
XMEAS $(15)$ & Stripper level \\
XMEAS $(16)$ & Stripper pressure \\
XMEAS $(17)$ & Stripper underflow \\
XMEAS $(18)$ & Stripper temperature \\
XMEAS $(19)$ & Stripper steam flow \\
XMEAS(20) & Compressor work \\
XMEAS $(21)$ & Reactor CW outlet temperature \\
XMEAS(22) & Condenser CW outlet temperature \\
\hline
\end{tabular}

Table 2 Faults

\begin{tabular}{lll}
\hline Number & Process variable & Type \\
\hline IDV(1) & A/C feed ratio & Step \\
IDV(2) & B composition & Step \\
IDV(3) & D feed temperature & Step \\
IDV(4) & Reactor cooling water inlet temperature & Step \\
IDV(5) & Condenser cooling water inlet & Step \\
& temperature & \\
IDV(6) & A feed loss & Step \\
IDV(7) & C header pressure loss & Step \\
IDV(8) & A,B,C feed composition & Random \\
IDV(9) & D feed temperature & Random \\
IDV(10) & C feed temperature & Random \\
IDV(11) & Reactor cooling water inlet temperature & Random \\
IDV(12) & Condenser cooling water inlet & Random \\
& temperature & \\
IDV(13) & Reaction kinetics & Slow drift \\
\hline
\end{tabular}

limits. Figure 2 shows the XmR charts of these two projections. Figure 2(a) is on the optimal discriminant direction from all the 22 continuous measurements where the last two samples are out of the control limits. The contributions for three variables, XMEAS(1), XMEAS(10) and XMEAS(21), exceeded to 0.1 $w_{\max }$ and these variables were selected as the key variables. Figure 2(b) shows the XmR chart on the optimal discriminant direction from the selected three variables. As shown in this figure, all the samples are within the control limits. From this comparison, variable selection can increase the performance of fault detection.

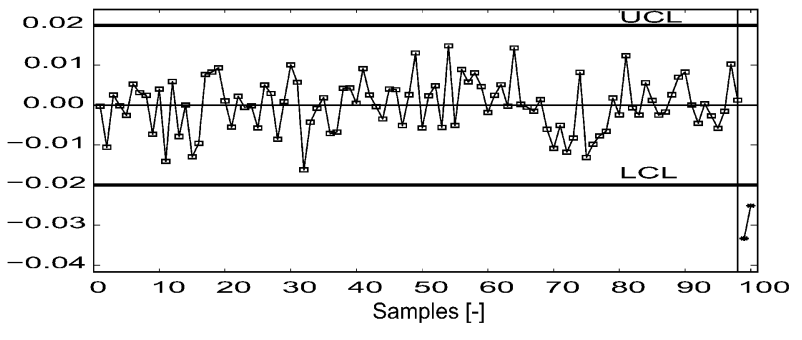

(a) Using all the variables

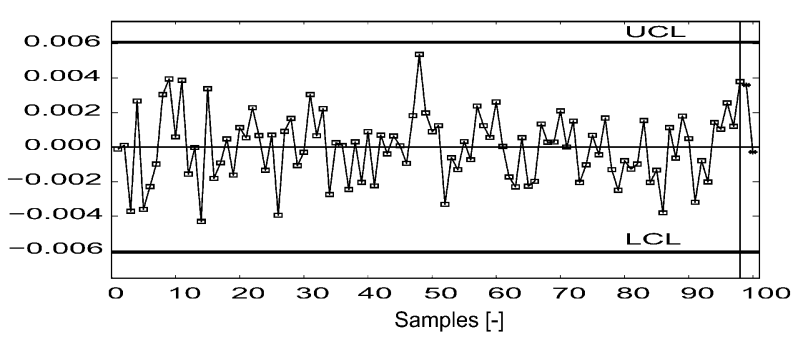

(b) Using selected variables

Fig. 2 Examples of XmR chart on FDA direction

\subsection{Fault detection}

Twenty five cases of time series data were generated for each faults by combining five runs of normal and abnormal cases. Each case includes 100 normal samples and 100 abnormal samples. For comparison, conventional XmR control charts on original measurement variables were calculated. Figure 3 shows several examples of the charts for a data set including IDV(3) fault. The corresponding control limits calculated by Eqs. (15) and (16) are also shown in the figure. Several variables like XMEAS(9) exceed the control limits but the delay of the detection was more than 20 samples. Most of the other measurement variables can not detect the fault. Now, the proposed method was applied to the same data set. By projecting these original signals on the FDA direction, control chart of this projected variable can be drawn as Figure 4. In this chart the fault was easily detected.

The algorithm of fault detection was applied to these 25 cases for each faults. Figure 5 shows an example of the set of the FDA-XmR chart for monitoring IDV(3). Current data in Figure 5(a) is the first two samples just after the occurrence of the fault. Current data in Figure 5(b) are the second and the third samples after the occurrence of the fault. Because the discriminant direction changes dynamically, shape and control limits of the chart change dynamically. In this time series, the fault is detected in Figure 5(b), where both of the two successive samples exceed the control limits. Table 3 summarises the result in terms of the average run length (ARL). Run length (RL) is the number of samples between the occurrence and the detection of a fault and the ARL is the average of the 

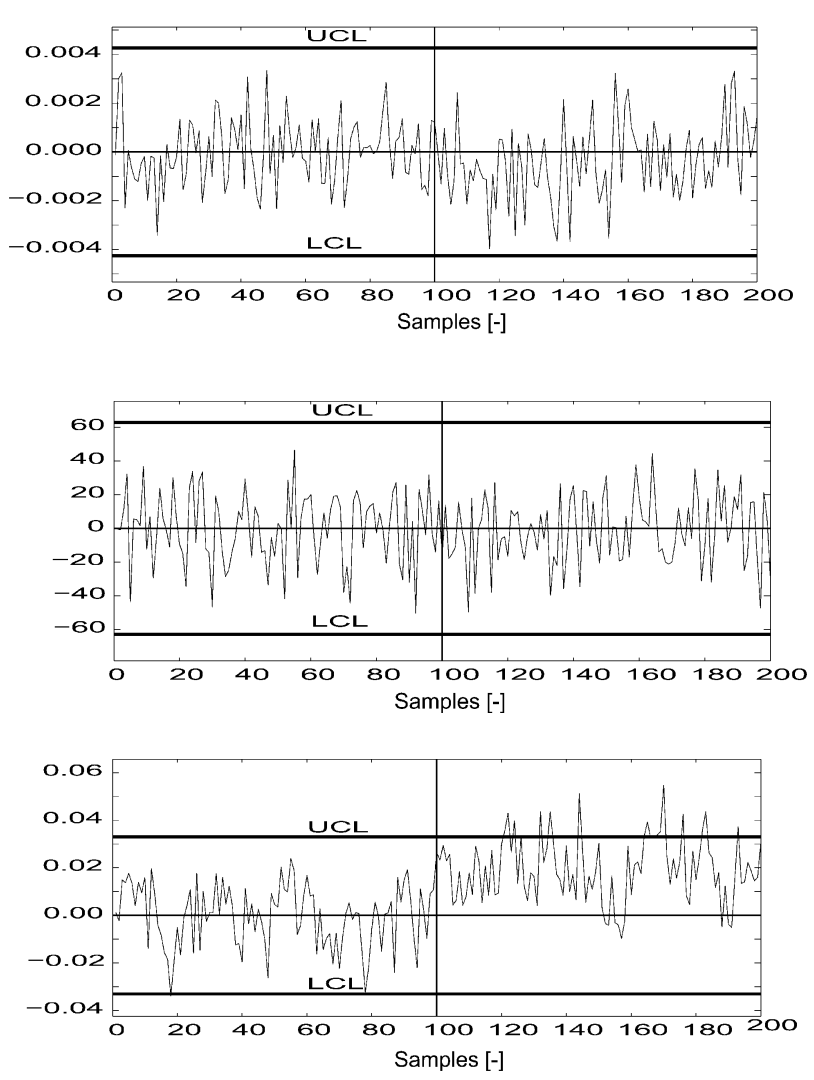

Fig. 3 Several examples of control chart on the original signals

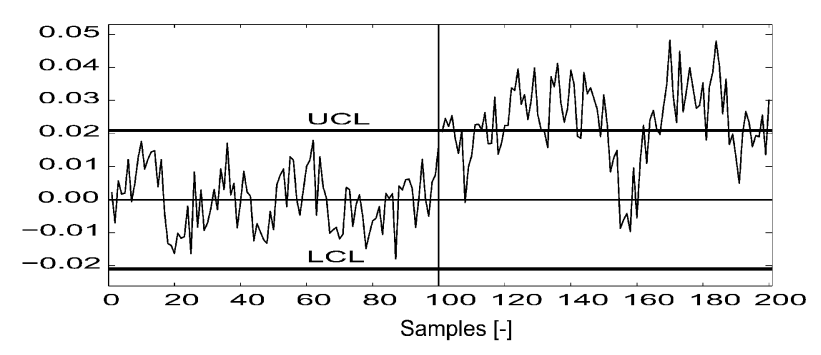

Fig. 4 Control chart on FDA direction

RL of 25 detections. Smaller value of the ARL indicates that the fault can be detected within a short delay. Numbers in the second column on the table are the ARLs by the proposed FDA-XmR method. All the step types of faults show smaller ARLs than the other faults, it means that these faults were detected faster than the others. Slow drift shows the largest ARL, it means that this type of faults is most difficult to detect.

To compare the performance of the proposed method, two other conventional methods using PCAbased $T^{2}$ statistics and PCA-based $Q$ statistics were applied to the same data sets (Figure 6). Table 3 also includes these results, where the first two principal components were used in these calculations. These two methods detected most step types of faults almost as
Table 3 Comparison of the ARL

\begin{tabular}{lccr}
\hline IDV No. & FDA-XmR & PCA- $Q$ & PCA- $T^{2}$ \\
\hline IDV(1) & 1 & 1 & 1.92 \\
IDV(2) & 2 & 2.8 & 3.96 \\
IDV(3) & 4.16 & 17.6 & 23.92 \\
IDV(4) & 1 & 1 & 1.12 \\
IDV(5) & 1.04 & 1.04 & 1.36 \\
IDV(6) & 1 & 1 & 1 \\
IDV(7) & 1 & 1 & 1 \\
IDV(8) & 10.36 & 10.2 & 11.16 \\
IDV(9) & 14.56 & 23.12 & 32.32 \\
IDV(10) & 12.32 & 15.04 & 17.24 \\
IDV(11) & 6.56 & 7.28 & 8.4 \\
IDV(12) & 6.04 & 6.72 & 7.08 \\
IDV(13) & 19.68 & 23.68 & 25.72 \\
\hline
\end{tabular}

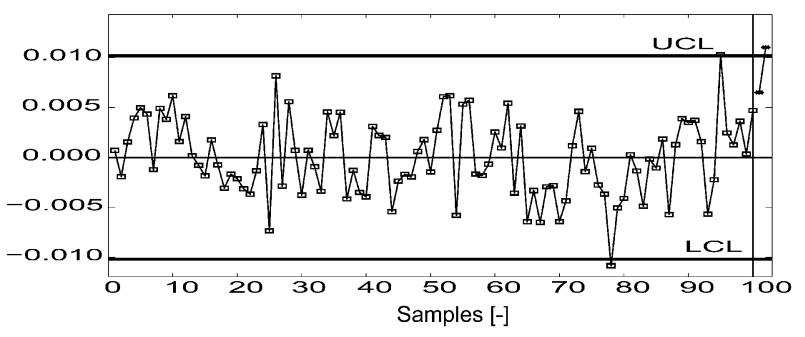

(a) Just after the occurrence of the fault

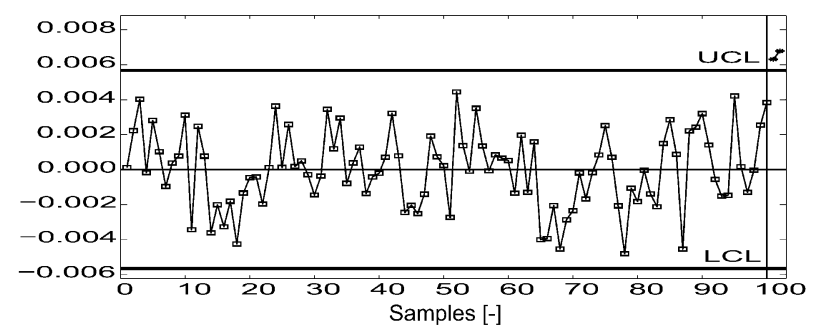

(b) At the next sample

Fig. 5 Control charts for IDV(3) by FDA-XmR

fast as the FDA-XmR, but they can not detect other faults faster than the FDA-XmR method. The results show the advantage of the FDA-XmR method over PCA- $Q$ and PCA- $T^{2}$ methods. Among them, drastic advantage of the proposed method was shown in step faults IDV(3) and random fault IDV(9).

\section{Conclusions}

A new statistical process control method has been proposed by combining the classification performance of FDA with the quality control advantage of control charts. The contribution plot based on the optimal discriminant direction was also included in the methodology to reduce the number of variables and improve 

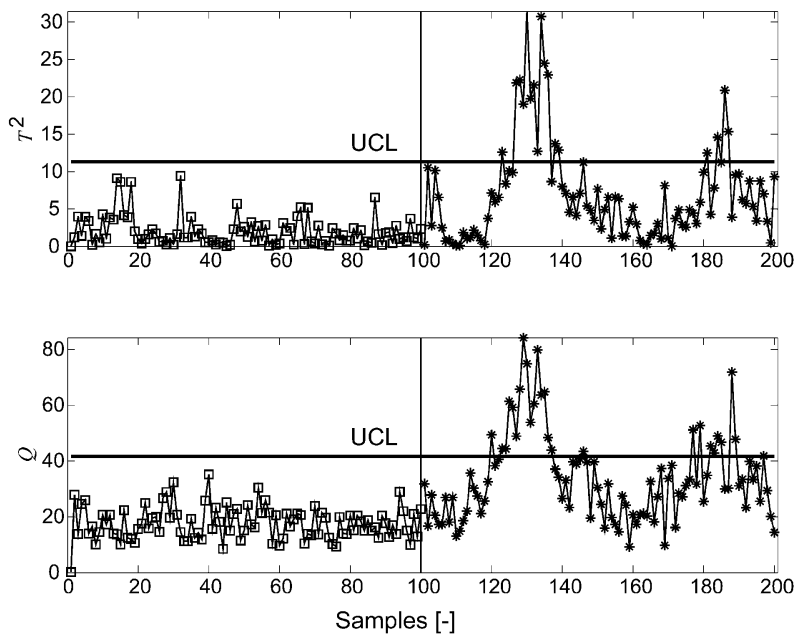

Fig. 6 Control charts for IDV(3) by PCA

the detection performance. The method was applied to the Tennessee Eastman process and compared with conventional PCA methods based on $Q$ and $T^{2}$ statistics. The results showed that the fault detection performance of the proposed method was the best among these three methods.

\section{Literature Cited}

Bakshi, B. R.; "Multiscale PCA with Application to Multivariate Statistical Process Monitoring," AIChE J., 44, 1596-1610 (1998)

Chen, G. and S. W. Cheng; "Max-Chart: Combining X-Bar Chart and S Chart," Statistica Sinica, 8, 263-271 (1998)

Chen, G., S. W. Cheng and H. Xie; "Monitoring Process Mean and Variability with One EWMA Chart," J. Qual. Technol., 33, 223233 (2001)

Chen, G., S. W. Cheng and H. Xie; "A New EWMA Control Chart for Monitoring both Location and Dispersion," Quality Technol. Quant. Manag., 1, 217-231 (2004)

Chen, J. and K.-C. Liu; "Online Batch Process Monitoring Using Dynamic PCA and Dynamic PLS Models," Chem. Eng. Sci., 57, 63-75 (2002)

Cheng, S. W. and K. Thaga; "Single Variables Control Charts: An Overview," Qual. Reliab. Eng. Int., 22, 811-820 (2006)

Chiang, L. H. and R. J. Pell; "Genetic Algorithms Combined with Discriminant Analysis for Key Variable Identification," J. Process Control, 14, 143-155 (2004)

Chiang, L. H., E. L. Russell and R. D. Braatz; "Fault Diagnosis in Chemical Processes Using Fisher Discriminant Analysis, Discriminant Partial Least Squares, and Principal Component Analysis," Chemom. Intell. Lab. Syst., 50, 243-252 (2000)

Cho, J.-H., J.-M. Lee, S. W. Choi, D. Lee and I.-B. Lee; "Fault Identification for Process Monitoring Using Kernel Principal Component Analysis," Chem. Eng. Sci., 60, 279-288 (2005)

Crosier, R. B.; "Multivariate Generalizations of Cumulative Sum
Quality-Control Schemes,” Technometrics, 30, 291-303 (1988)

Downs, J. J. and E. F. Vogel; "A Plant-Wide Industrial Process Control Problem," Comput. Chem. Eng., 17, 245-255 (1993)

Gan, F. F.; "Joint Monitoring of Process Mean and Variance," Nonlinear Anal., 30, 4017-4024 (1997)

He, Q. P., S. J. Qin and J. Wang; "A New Fault Diagnosis Method Using Fault Directions in Fisher Discriminant Analysis," AIChE $J ., 51,555-571(2005)$

Hotelling, H.; "Multivariate Quality Control," Techniques of Statistical Analysis, C. Eisenhart, M. W. Hastay and W. A. Wallis eds., pp. 111-184, McGraw-Hill, New York, U.S.A. (1947)

Kresta, J. V., J. F. MacGregor and T. E. Marlin; "Multivariate Statistical Monitoring of Process Operating Performance," Can. J. Chem. Eng., 69, 35-47 (1991)

Ku, W., R. H. Storer and C. Georgakis; "Disturbance Detection and Isolation by Dynamic Principal Component Analysis," Chemom. Intell. Lab. Syst., 30, 179-196 (1995)

Lee, J.-M., C. K. Yoo, S. W. Choi, P. A. Vanrolleghem and I. B. Lee; "Nonlinear Process Monitoring Using Kernel Principal Component Analysis," Chem. Eng. Sci., 59, 223-234 (2004)

Li, W., H. Yue, S. Valle-Cervantes and S. J. Qin; "Recursive PCA for Adaptive Process Monitoring," J. Process Control, 10, 471486 (2000)

Lowry, C. A., W. H. Woodall, C. W. Champ and S. E. Rigdon; "A Multivariate Exponentially Weighted Moving Average Control Chart," Technometrics, 34, 46-53 (1992)

McAvoy, T. J. and N. Ye; "Base Control for the Tennessee Eastman Problem," Comput. Chem. Eng., 18, 383-413 (1994)

Miller, P., R. E. Swanson and C. F. Heckler; "Contribution Plots: A Missing Link in Multivariate Quality Control," Appl. Math. Comput. Sci., 8, 775-792 (1998)

Nomikos, P. and J. MacGregor; "Monitoring Batch Processes Using Multiway Principal Component Analysis," AIChE J., 40, 1361-1375 (1994)

Page, E. S.; "Continuous Inspection Schemes," Biometrika, 41, 100$115(1954)$

Pignatiello, J. J., Jr. and G. C. Runger; "Comparisons of Multivariate CUSUM Charts,” J. Qual. Technol., 22, 173-186 (1990)

Qin, S. J.; "Statistical Process Monitoring: Basics and Beyond," J. Chemom., 17, 480-502 (2003)

Roberts, S. W.; "Control-Charts-Tests Based on Geometric Moving Averages," Technometrics, 1, 239-250 (1959)

Schölkopf, B., A. J. Smola and K.-R. Müller; "Nonlinear Component Analysis as a Kernel Eigenvalue Problem," Neural Computation, 10, 1299-1399 (1998)

Shewhart, W. A.; Economic Control of Quality of Manufactured Product, D. van Nostrand Company, Toronto, Canada (1931)

Smith, G. M.; Statistical Process Control and Quality Improvement, 4th ed., Prentice Hall, Uppel Saddle River, U.S.A. (2001)

Spiring, F. A. and S. W. Cheng; "An Alternate Variables Control Chart: The Univariate and Multivariate Case," Statistica Sinica, 8, 273-287 (1998)

Thaga, K.; Contributions to Statistical Process Control Tools, Ph.D. Thesis, University of Manitoba, Winnipeg, Canada (2003)

Wang, D. and J. A. Romagnoli; "Robust Multi-Scale Principal Components Analysis with Applications to Process Monitoring," J. Process Control, 15, 869-882 (2005)

Xie, H.; Contributions to Qualimetry, Ph.D. Thesis, University of Manitoba, Winnipeg, Canada (1999) 